\title{
An Assessment of the Nutritional Status of Students in Governmental Primary Schools in U.A.Q.
}

\author{
Hanan Soliman', Shaza Shaaban Zackaria², and Hiba Rabie Mohammed ${ }^{3}$ \\ ${ }^{1}$ Senior Charge Tech. in Dental health center/U.A.Q./MOHAP \\ ${ }^{2}$ Family Physician in PHCC /U.A.Q. /MOHAP \\ ${ }^{3}$ General Practitioner/Family PhysicianPHCC /U.A.Q. /MOHAP
}

Corresponding Author: Shaza Shaaban Zackaria; Mailing address: P.O. Box 62772, Dubai,U.A.E. email:

shazashaaban@gmail.com Telephone: 00971566272621

Received: 12 March 2019

Accepted: 24 April 2019

Published: 30 April 2019

Production and Hosting by Knowledge E

(c) Hanan Soliman et al. This article is distributed under the terms of the Creative

Commons Attribution

License, which permits

unrestricted use and

redistribution provided that the original author and

source are credited.

Editor-in-Chief:

Dr. Dimitrios Papandreou

Official Publication of Zayed University, UAE

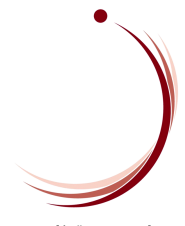

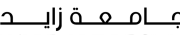
ZAYED UNIVERSITY

G OPEN ACCESS

\section{Abstract}

Aim: To provide a summary of the recent data on the nutritional status of school-aged children in developing countries and countries in transition and identify issues of public health concern. An assessment of the nutritional status of students in governmental primary schools in U.A.Q. Methods: A retrospective study was conducted from 2016 to 2017 on the nutrition status of Grade 1 school children in Government primary schools. The data was collected from questionnaires that were distributed routinely. Results: A total of 317 students from 7 different primary public schools in UAQ, with an age range from 5-7 years. Students with Chronic diseases, congenital anomalies, and hereditary diseases were excluded. The available data indicate that the nutritional status of the students in the reviewed schools is adequate. Where it has shown that mean body mass index (BMI) was 15.2. Underweight, Overweight obesity was not prominent with a percentage of $12 \%$ and $10 \%$ respectively. As for anemia, only $7 \%$ were diagnosed biochemically. With the mean hemoglobin of $12.1 \mathrm{mg} / \mathrm{dl}$ overall. Conclusions: The available data indicate that malnutrition is not a health issue in primary students in UAQ primary public schools. These findings emphasize the impact of the school health program on primary students.

\section{Introduction}

The nutritional status of school-aged children: Why should we care?

Nutritional status of primary school children is important to assess and evaluate to assist in developing a school feeding program which promotes improved health status during this age. Poor nutritional status has a negative effect on health and developmental status which will have an adverse effect on their school performance and will render them prone to developing chronic diseases later on. There are not enough studies to overlook the nutritional status of primary school children.

It has been recognized by several organizations and governments that amelioration of health and nutrition of primary school students, has positively contributed to their educational achievements, growth, and development. Hence, schools are practical grounds in providing interventions such as; healthy meals and life skills education. Researches and data on the actual nutritional status of school children have been 
lacking in developing countries and countries in transition. It has been stated that school-aged children are excluded from nutrition surveillance.

It is evident that introducing nutritional care programs and other interventions to children (2-3 years) had a better impact on nutritional and developmental status than when done at older ages (5-6 years). Furthermore, the WHO and World Bank have stated that early child development programs have a long-term impact in reducing poverty. Thus, it is important to assess that nutritional status of school-aged children as they might be at risk of weak growth and development. (Best et al. 2010)

Health authorities in several Arab countries are developing a growing concern towards unhealthy lifestyle and poor dietary habits, as obesity have been on the rise and have reached an epidemic rate in children.

According to the Global School Health Survey (GSHS) 2010, in the United Arab Emirates (UAE) approximately $40 \%$ of schoolchildren are overweight or obese. Other than being physically unwell, obesity makes children target to discrimination and stereotyping, hence overweight and obesity affects a child's social, academic and mental health negatively. (Haroun et al. 2017)

Nutrition is very important in determining a body response to factor that can cause disease if not resisted. No treatment will be totally effective if the nutritional state of the student is inadequate or unbalanced (Best et al. 2010).

Given this, there is a need to determine the relationship between the nutritional statuses of children less than 6 years of age and the occurrence of various disease and anemia in childhood in UAQ. data generated from this study will help in building a body of knowledge about the nutritional status of children in UAQ schools. This kind of knowledge is required for all healthcare personnel who provide care for children, especially for the school nurse. It can help him/her determine the nutritional needs of these children in relation to assessment and provision of nutritional care

\section{Method}

A retrospective study that was conducted from 2016 to 2017 observing the nutritional status of school children in Grade 1 in 8 Government primary schools in Umm al Qwuain. Data was collected from questionnaires that were distributed routinely. In addition to a consent form that was signed by the parents. The questionnaire constituted of 3 parts and were distributed out to the students' parents. Where the parents were required to fill the first 2 parts of it regarding the feeding patterns, the socioeconomic status and the nutritional habits of the children. Whereas, the school nurse filled the last part of the questionnaire regarding the physical assessment that is annually conducted by a visiting school health doctor. The inclusion criteria was for all students attending primary Governmental schools in UAQ/UAE. The exclusion criteria was for children suffering of any congenital or chronic diseases. An ethical approval was obtained from the Ethical committee of UAQ medical district.

Descriptive statistics were used to describe the socio-demographic characteristics of the study participants. Mean (standard deviations, SD), Median and mode were reported 
for continuous variables. Frequencies with percentages were reported for categorical variables.

The analysis was performed using Statistical Package for Social Science (SPSS), version 23 (IBM, Armonk, NY, USA)

\section{Results}

TABLE 1: socio-demographic characteristics of the participants, $n$ (\% or SD).

\begin{tabular}{|c|c|}
\hline \multicolumn{2}{|l|}{ Gender } \\
\hline Male & $136(42.9)$ \\
\hline Female & $181(57.1)$ \\
\hline \multicolumn{2}{|l|}{ Age category, years } \\
\hline $5-6$ & $238(75.1)$ \\
\hline $6.1-7$ & 79 (24.1) \\
\hline \multicolumn{2}{|l|}{ Nationality } \\
\hline Local & $274(86.4)$ \\
\hline Non-local & $43(13.6)$ \\
\hline \multicolumn{2}{|c|}{ Socio-economic Status } \\
\hline High & 45 (14.2) \\
\hline Medium & $222(70)$ \\
\hline Low & $50(15.8)$ \\
\hline \multicolumn{2}{|l|}{ Father's Education } \\
\hline Primary & $43(13.6)$ \\
\hline Secondary & $204(64.3)$ \\
\hline Tertiary & $70(22.1)$ \\
\hline \multicolumn{2}{|l|}{ Mother's Education } \\
\hline Primary & $41(13)$ \\
\hline Secondary & 199 (63) \\
\hline Tertiary & $76(24)$ \\
\hline \multicolumn{2}{|l|}{ Child's order } \\
\hline $1^{s t}-2^{n d}$ & 109 (34.4) \\
\hline $3^{r d}-4^{\text {th }}$ & $146(46)$ \\
\hline$\geq 5^{t h}$ & 62 (19.6) \\
\hline \multicolumn{2}{|c|}{ Body mass index (BMI), $\mathrm{kg} / \mathrm{m}^{2}$} \\
\hline Mean (SD) & $15.2(2.9)$ \\
\hline \multicolumn{2}{|c|}{ Haemoglobin (Hb), mg/dl } \\
\hline Mean (SD) & $12.1(0.8)$ \\
\hline \multicolumn{2}{|c|}{ Mean Corpuscular Volume (MCV), fL/red } \\
\hline Mean (SD) & $73.6(12.3)$ \\
\hline \multicolumn{2}{|c|}{ Mean Corpuscular Hemoglobin (MCH), (pg)/cel } \\
\hline Mean (SD) & $26.4(8.5)$ \\
\hline \multicolumn{2}{|c|}{ Body Mass Index (BMI) category, kg/m² } \\
\hline Underweight $(\leq 13)$ & $38(12)$ \\
\hline Normal (13.1-17) & $217(68.5)$ \\
\hline Overweight (17.1-19) & $29(9.1)$ \\
\hline Obese $(\geq 19.1)$ & $33(10.4)$ \\
\hline
\end{tabular}


In Table 1. students included were 136 males and 181 females with the majority being from 5-6 years old (75.1\%). 86.4\% were Emirati with 70\% from a middle socioeconomic class. Most of the parents of these students had completed secondary education. The mean body mass index was $15.2 .12 \%$ were underweight, $68.5 \%$ had a normal weight. Around $10 \%$ of these students were overweight and obese.When investigating students for anemia, only $7 \%$ were diagnosed biochemically (Figure 2 ). With the mean hemoglobin of $12.1 \mathrm{mg} / \mathrm{dl}$ overall (Table 1).

The percentage of overweight was slightly increased in the 5-6 age group, while the obesity percentage was slightly higher in the 6-7 age group. The underweight percentage was higher in the 5-6 age group and the same was for anemia in this age group. (Table 2)The percentage of obesity was higher in Female students while the percentage of overweight and underweight were higher in the male students. The number of anemic females was more than double the number of anemic male students. (Table 2)Most of the students in all socioeconomic statuses were having a normal BMI. Percentage of underweight was $15.6 \%$ and $12 \%$ in the high and medium socioeconomic status respectively. $10 \%$ were obese and overweight among the low socioeconomic class. (Table 2)

The relation between education and weight

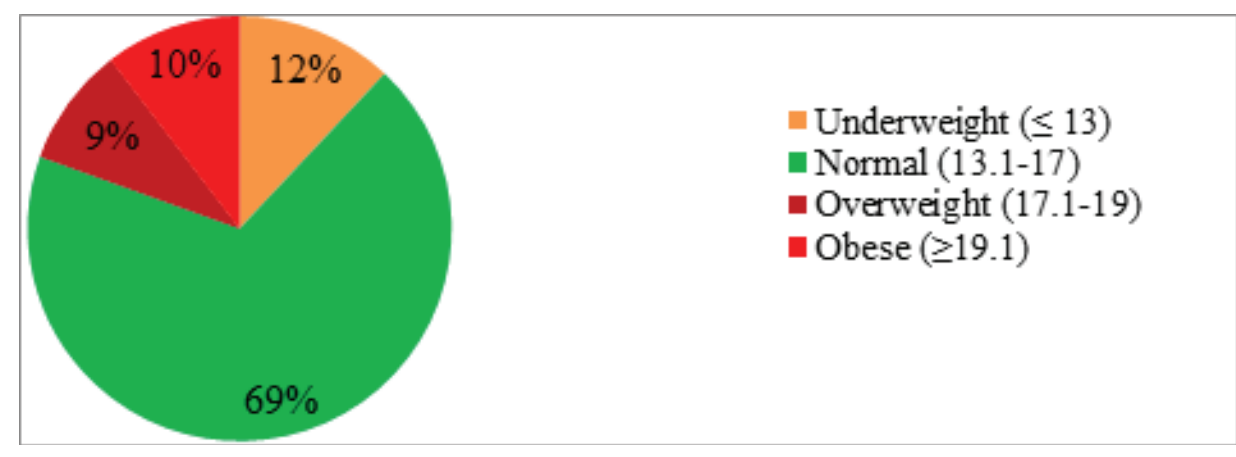

Figure 1: Pie chart showing the overall BMI distribution for children.

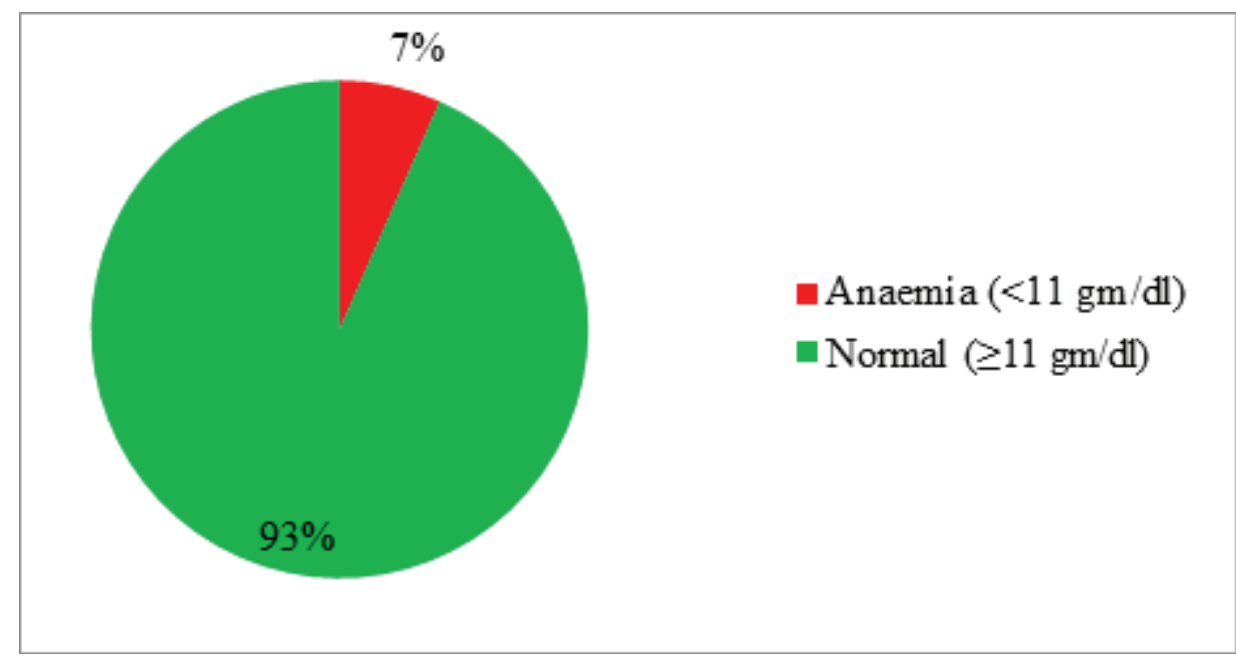

Figure 2: Pie chart showing the overall anemia status for children. 
TABLE 2: Distribution of BMI categories and anemia status by age, gender, and SES

\begin{tabular}{|c|c|c|c|c|c|c|}
\hline \multirow[t]{2}{*}{ Variable } & \multicolumn{4}{|c|}{ BMI } & \multicolumn{2}{|c|}{ Anemia } \\
\hline & $\begin{array}{l}\text { Normal } \\
\left(13.1-17 \mathrm{~kg} / \mathrm{m}^{2}\right)\end{array}$ & $\begin{array}{l}\text { Under weight } \\
\left(\leq 13 \mathrm{~kg} / \mathrm{m}^{2}\right)\end{array}$ & $\begin{array}{l}\text { Over weight } \\
\left(17.1-19 \mathrm{~kg} / \mathrm{m}^{2}\right)\end{array}$ & $\begin{array}{c}\text { Obese } \\
\left(\geq 19.1 \mathrm{~kg} / \mathrm{m}^{2}\right)\end{array}$ & Yes & No \\
\hline \multicolumn{7}{|l|}{ Age, years } \\
\hline $5-6$ & $160(67.2)$ & $31(13)$ & $23(9.7)$ & $24(10.1)$ & 17 (7.1) & 221 (92.9) \\
\hline $6.1-7$ & 57 (72.1) & $7(8.9)$ & $6(7.6)$ & $9(11.4)$ & $4(5.1)$ & 75 (94.9) \\
\hline \multicolumn{7}{|l|}{ Gender } \\
\hline Male & $93(68.4)$ & $20(14.7)$ & $13(9.6)$ & $10(7.4)$ & $6(4.4)$ & 130 (95.6) \\
\hline Female & $124(68.5)$ & $18(10)$ & $16(8.8)$ & $23(12.7)$ & $15(8.3)$ & $166(91.7)$ \\
\hline \multicolumn{7}{|c|}{ Socio-economic Status (SES) } \\
\hline High & $30(66.7)$ & 7 (15.6) & $3(6.7)$ & $5(11)$ & $3(6.7)$ & 42 (93.3) \\
\hline Medium & $151(68)$ & $27(12.2)$ & $21(9.4)$ & $23(10.4)$ & $14(6.3)$ & 208 (93.3) \\
\hline Low & $36(72)$ & $4(8)$ & $5(10)$ & $5(10)$ & $4(8)$ & $46(92)$ \\
\hline \multicolumn{7}{|l|}{ Child order } \\
\hline $1^{s t}-2^{n d}$ & 71 (65.1) & $16(14.7)$ & $13(11.9)$ & $9(8.3)$ & $8(7.3)$ & $101(92.7)$ \\
\hline $3^{r d}-4^{t h}$ & $100(68.5)$ & $14(9.6)$ & $14(9.6)$ & $18(12.3)$ & $6(4.1)$ & 140 (95.9) \\
\hline$\geq 5^{t h}$ & $46(74.2)$ & 8 (12.9) & $2(3.2)$ & $6(9.6)$ & 7 (11.3) & $55(88.7)$ \\
\hline \multicolumn{7}{|c|}{ Father's Education } \\
\hline Primary & $32(74.4)$ & $3(7.0)$ & $2(4.7)$ & $6(14.0)$ & 5 (11.6) & 38 (88.4) \\
\hline Secondary & $140(68.6)$ & $26(12.7)$ & $21(10.3)$ & $17(8.3)$ & $13(6.4)$ & 191 (93.6) \\
\hline Tertiary & $46(64.3)$ & $9(12.9)$ & $6(8.6)$ & $10(14.3)$ & $3(4.3)$ & $67(95.7)$ \\
\hline \multicolumn{7}{|c|}{ Mother's Education } \\
\hline Primary & $28(68.3)$ & $6(14.6)$ & $3(7.3)$ & $4(9.8)$ & $3(7.3)$ & $38(92.7)$ \\
\hline Secondary & $139(69.8)$ & $21(10.6)$ & $19(9.5)$ & $20(10.1)$ & $16(8.0)$ & $183(92.0)$ \\
\hline Tertiary & $49(64.5)$ & $11(14.5)$ & $7(9.2)$ & 9 (11.8) & $2(2.6)$ & $74(97.4)$ \\
\hline
\end{tabular}

In Table 2. it has shown that $14.6 \%$ of those whom their mother education is up to primary school are underweight, $10 \%$ of those with maternal secondary education are underweight and the same percentage for those who are obese. Students of mothers of higher education are $14.5 \%$ under weight and only $2.6 \%$ of them are anemic. (Table 2)

Table 3. Regarding the number of meals per day, $13 \%$ of those who have 1 to 2 meals per day are obese and around 7\% are anemic. Those who have four meals per day; $17 \%$ are obese and $11 \%$ are anemic. A percentage of 81.6 responded that the students have regular breakfast, around $9 \%$ of those are obese and $7 \%$ are anemic. Those who don't have regular breakfast were $18.4 \%$, around $7 \%$ are anemic and $17 \%$ are obese. Most of the students have beverages between meals and among them, $7 \%$ are anemic and $10.4 \%$ are obese. Students who are used to take snacks between meals; $8.2 \%$ of them are anemic and $10 \%$ are obese. (Table 3 )

Table 4 shows the mean, median and mode of the students' anthropometric measurement in both males and females. There was a slight difference between both genders. 
TABLE 3: Distribution of anemia and obesity by eating habits, $n(\%)$

\begin{tabular}{|l|c|c|c|}
\hline $\begin{array}{l}\text { Eating habits } \\
\text { Number of meals per day }\end{array}$ & Total & Anemia & Obesity \\
\hline $1-2$ & $15(4.7)$ & $1(6.7)$ & $2(13.3)$ \\
\hline 3 & $266(84.2)$ & $16(6)$ & $25(9.4)$ \\
\hline 4 & $35(11.1)$ & $4(11.4)$ & $6(17.1)$ \\
\hline Taking breakfast regularly & & & $23(8.9)$ \\
\hline Yes & $257(81.6)$ & $17(6.6)$ & $10(17.2)$ \\
\hline No & $58(18.4)$ & $4(6.9)$ & $31(10.4)$ \\
\hline Beverage between meals & & & $2(11.1)$ \\
\hline Yes & $299(94.3)$ & $21(7)$ & \\
\hline No & $18(5.7)$ & $0(0)$ & $23(10)$ \\
\hline Snacks between meals & & $19(8.2)$ & $10(11.6)$ \\
\hline Yes & $231(82.9)$ & $2(2.3)$ & \\
\hline No & $86(27.1)$ & & \\
\hline
\end{tabular}

TABLE 4: Mean, median and mode of anthropometrics measurement by gender.

$\begin{aligned} & \text { Anthropometrics } \\ & \text { measurement }\end{aligned}$
Weight $(\mathrm{kg})$
Height $(\mathrm{m})$
BMI $\left(\mathrm{kg} / \mathrm{m}^{2}\right)$
Head circumference $(\mathrm{cm})$
Arm circumference $(\mathrm{cm})$
Chest circumference $(\mathrm{cm})$

\begin{tabular}{|c|c|}
\hline \multicolumn{2}{|c|}{ Mean } \\
\hline Male & Female \\
\hline 21.3 & 20.6 \\
\hline 1.19 & 1.16 \\
\hline 15.1 & 15.3 \\
\hline 52.9 & 52.2 \\
\hline 18.2 & 18 \\
\hline 59.4 & 59.7 \\
\hline
\end{tabular}

\begin{tabular}{|c|c|}
\hline \multicolumn{2}{|c|}{ Median } \\
\hline Male & Female \\
\hline 20 & 19 \\
\hline 1.18 & 1.15 \\
\hline 14.6 & 14.6 \\
\hline 52 & 51 \\
\hline 18 & 17 \\
\hline 59 & 58 \\
\hline
\end{tabular}

\begin{tabular}{|c|c|}
\hline \multicolumn{2}{|c|}{ Mode } \\
\hline Male & Female \\
\hline 18 & 17 \\
\hline 1.18 & 1.14 \\
\hline 13.4 & 13.1 \\
\hline 52 & 52 \\
\hline 18 & 17 \\
\hline 56 & 56 \\
\hline
\end{tabular}

TABLE 5: Clinical Examination.

\begin{tabular}{|l|c|}
\hline Problems & $\mathrm{N}(\%)$ \\
\hline Teeth problems & $45(14.2)$ \\
\hline Skin problems & $7(2.2)$ \\
\hline Mental problems & $1(0.3)$ \\
\hline Face problem & $1(0.3)$ \\
\hline Nails problem & $0(0)$ \\
\hline
\end{tabular}

Table 5. has shown findings in clinical examination, $14 \%$ had teeth problems, around $2 \%$ had a skin problem, $0.3 \%$ only had mental and face problem and no students nails problems.

In table 6. Students in Alwatan School were mostly normal with $13.6 \%$ being underweight, $9.1 \%$ overweight and only $1.5 \%$ obese with all students having no anemia. Alabraq school had $67.3 \%$ normal BMI ,12.9\% underweight ,10.9\% obese and $8.9 \%$ overweight, only $9.9 \%$ were anemic . In khawla,Majed and Saeed bin Yazeed school, there were no 
TABLE 6

School
Alwatan
Khawla
Bint Alshati
Alabrag
Majed
Ahmed Bin Rashid
Saad Bin Yazeed
Um Alshuhada

\begin{tabular}{c}
$\begin{array}{c}\text { Normal } \\
\left(13.1-17 \mathrm{~kg} / \mathrm{m}^{2}\right)\end{array}$ \\
$50(75.8)$ \\
$5(62.5)$ \\
$21(84)$ \\
$68(67.3)$ \\
$14(82.4)$ \\
\hline $27(57.4)$ \\
$2(33.3)$ \\
$30(63.8)$ \\
\hline
\end{tabular}

$\begin{gathered}\text { Underweight } \\ \left(\leq 13 \mathrm{~kg} / \mathrm{m}^{2}\right)\end{gathered}$
$9(13.6)$
$0(0)$
$1(4)$
$13(12.9)$
$0(0)$
$11(23.4)$
$0(0)$
$4(8.5)$

\begin{tabular}{|c|}
$\begin{array}{c}\text { Overweight } \\
\left(17.1-19 \mathrm{~kg} / \mathrm{m}^{2}\right)\end{array}$ \\
\hline $6(9.1)$ \\
\hline $2(25)$ \\
$1(4)$ \\
\hline $9(8.9)$ \\
\hline $1(5.9)$ \\
\hline $4(8.5)$ \\
\hline $2(33.3)$ \\
\hline $4(8.5)$ \\
\hline
\end{tabular}

$\begin{gathered}\text { Obese } \\ \left(\geq 19.1 \mathrm{~kg} / \mathrm{m}^{2}\right)\end{gathered}$
$1(1.5)$
$1(12.5)$
$2(8)$
$11(10.9)$
$2(11.8)$
$5(10.6)$
$2(33.3)$
$9(19.1)$

underweight students. The percentage of anemia in these schools was $0 \%, 5.9 \%$, and $16.7 \%$ respectively. Around $90 \%$ of the rest of the schools were having no anemia.

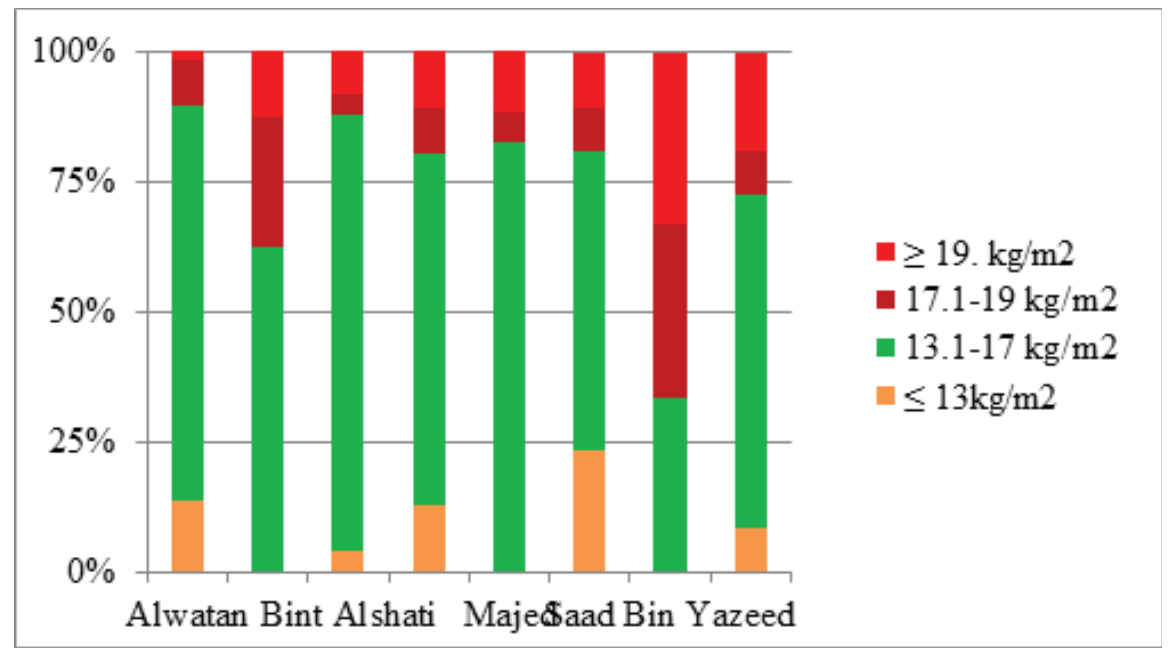

Figure 3

TABLE 7

School
Alwatan
Khawla
Bint Alshati
Alabrag
Majed
Ahmed Bin Rashid
Saad Bin Yazeed
Um alshuhada

\begin{tabular}{|c|c|}
\hline No anaemia & Anaemia \\
\hline $66(100)$ & $0(0)$ \\
\hline $8(100)$ & $0(0)$ \\
\hline $24(96)$ & $1(4)$ \\
\hline $91(90.1)$ & $10(9.9)$ \\
\hline $16(94.1)$ & $1(5.9)$ \\
\hline $43(91.5)$ & $4(8.5)$ \\
\hline $5(83.3)$ & $1(16.7)$ \\
\hline $43(91.5)$ & $4(8.5)$ \\
\hline
\end{tabular}




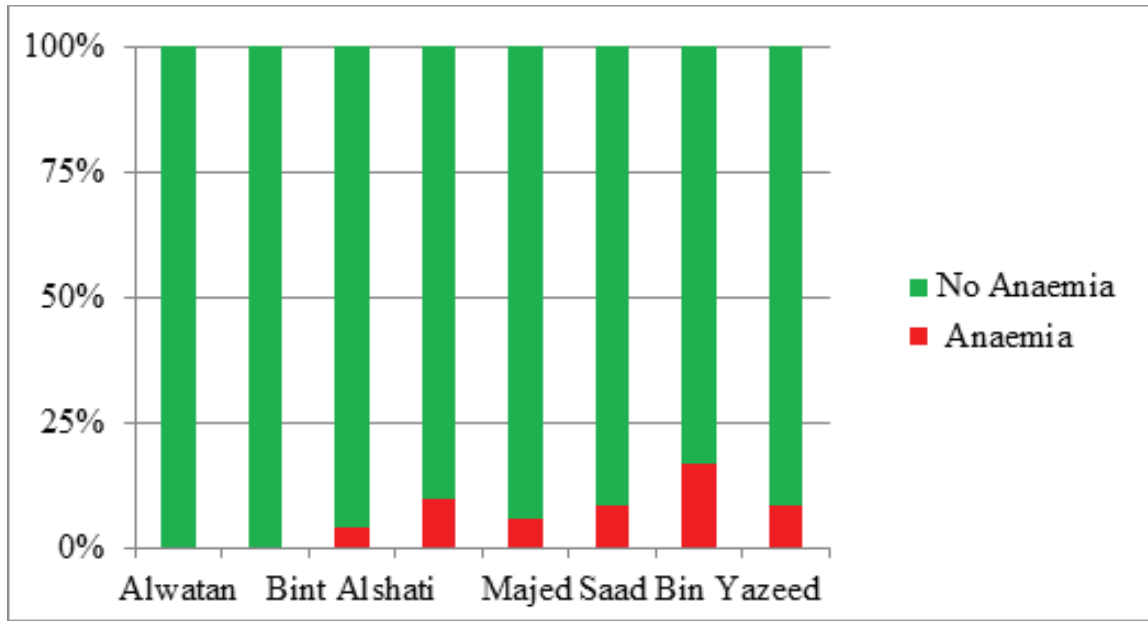

Figure 4

\section{Discussion}

Nutritional status of children was assessed in a field survey in selected emirates in the United Arab Emirates (UAE) where the anthropometric data collected for 1447 children aged 6-10 years from Emirati national population, Egyptian, Sudanese, Jordanian and Omani children living in the UAE. The results revealed that UAE children suffered from stunting and wasting in the study population. Whereas the comparison performed between children of the UAE and other nationalities living the UAE has shown that ethnic origin affected the centile and the Z-score distribution of the anthropometric indices. It was stated that the levels of malnutrition could be due to non-economic socio-cultural factors. (Hwalla et al.1997)

Lara et al (2018) reviewed the characteristics of the nutritional status of children in 4 countries which were Jordan, Lebanon, KSA, and UAE. They highlighted that the triple burden of malnutrition and poor dietary habits that could aid in understanding the nutritional status of children. It has also revealed the scarcity of studies reporting on dietary intakes and nutritional deficiencies in children, thus shedding light on the need for further efforts in the field of children's nutritional assessment in countries in this region.

It was stated that primary consideration should be provided to policies and interventions targeting the improvement of the nutritional status of children due to nutrition's impact on strengthening societies and transforming the lives of children and their families.

Galal and Hulett (2003) have focused in their paper on the association between children's nutritional status and their academic performance. Important determinants of school participation and school achievement are demographics and socio-economic characteristics of households. It was noted that undernourished children have expressed lower attendance, shorter attention span, lower performance scores and more health-related problems in comparison to their well-nourished peers. Childhood obesity has been investigated to assess the extent of its existence. It has been observed 
that children suffering the commonest symptoms of obesity such as tiredness, attention deficits, and hypertension lose the chance of healthy growth and succeeding at school.

As observed that educational levels of children could be affected, it has been determined that if children are approached during their early learning years positive impact will be achieved by decreasing risk factors and the promotion of healthy dietary habits. A shift in dietary habits has been realized from a traditional high-fiber diet to rice, processed wheat, and sugar consumption. Thus leading to diet associated diseases like diabetes, cardiovascular disease and cancer later in adulthood. The emphasis on the role of community and school physical exercises could show a reduction in the incidence of these diseases.

\section{Conclusion}

It has been recommended that prime concern should be drawn towards policies and interventions, which improve the nutritional status of the children, where they will tackle malnutrition along with achieving many ongoing developmental targets, which include; ensuring healthy lives, learning, and economic growth. (Lara et al. 2018) Whereas Ng et al (2011) have suggested that attention should be drawn towards educating young people especially females about nutrition and promoting physical activity. ( $\mathrm{Ng}$ et al. 2011)

Moreover, WHO (2000) stated that policymakers need to know that high rates of obesity in the population could inflict a burden on the national economic costs where they can range from $2 \%$ to $7 \%$ of the sum of health care costs. Therefore focusing on preventive programs on childhood obesity is cost-effective. (Sahota et al. 2001)

In this research, the results were insignificant hence not implying malnutrition in primary school children in UAQ Primary schools. This indicates that the school health program that is applied by UAQ Primary Health care Department is efficient and the plan has been successful to maintain a good nutritional status for primary school children in UAQ public school. We recommend a follow-up study of the nutritional status of the students to be able to rule out other factors, which could have an impact on their health.

\section{References}

[1] Lara M. Nasreddine, Amira N. Kassis, Jennifer J. Ayoub, Farah A. Naja, Nahla C. Hwalla, (2018) Nutritional status and dietary intakes of children amid the nutrition transition: the case of the Eastern Mediterranean Region, Nutrition Research. (57):12-27. ISSN 0271-5317,

[2] Ng, S. W., Zaghloul, S., Ali, H., Harrison, G., Yeatts, K., El, M., \& Popkin, B. M. (2011). Nutrition Transition in the United Arab Emirates (UAE). European Journal of Clinical Nutrition, 65(12), 1328-1337.

[3] Best C, Neufingerl N, van Geel L, van den Briel T, Osendarp S. (2010)The nutritional status of schoolaged children: why should we care? Food Nutr Bull.31(3):400-17. Review. PubMed PMID: 20973461.

[4] Osman Galal and Judie Hulett (2003) The relationship between nutrition and children's educational performance: a focus on the United Arab Emirates. British Nutrition Foundation Nutrition Bulletin, 28, $11-20$

[5] Haroun, D., EISaleh, O., \& Wood, L. (2017). Dietary and Activity Habits in Adolescents Living in the United Arab Emirates: A Cross-Sectional Study. Arab Journal of Nutrition and Exercise (AJNE), 1(2), 85-100. 
[6] Hwalla, Nahla \& Shaar, Khuzama \& Faour-Klingbeil, Dima \& Musaiger, Abdulrahman \& Adra, Nada. (1997). Nutritional status of school children aged 6-10 years in the United Arab Emirates: Comparison with children from different ethnic origins. Ecology of Food and Nutrition - ECOL FOOD NUTR. 36. 367-384. 10.1080/03670244.1997.9991525. 